\title{
Des YAC dans des souris transgéniques
}

Les chromosomes artificiels de levure (YAC) sont de plus en plus utilisés par les biologistes moléculaires pour établir la carte de grandes régions chromosomiques aussi bien que pour isoler des loci ou des gènes complexes. L'efficacité avec laquelle la recombinaison homologue est réalisée chez la levure permet de modifier par ce moyen des gènes ou des séquences régulatrices localisées sur un YAC. Il était donc très tentant d'utiliser directement un tel matériel pour créer des souris transgéniques portant des gènes normaux ou modifiés. Une difficulté rencontrée jusqu'alors était cependant d'obtenir suffisamment de matériel pour pouvoir réaliser les expériences aboutissant à la création de lignées de souris transgéniques. Trois équipes, l'une de Californie [1] une autre de Heidelberg, en Allemagne [2] et une troisième du MIT (Cambridge, MA, USA) [3] viennent de rapporter la création de telles lignées de souris transgéniques. La première équipe a fusionné des sphéroplastes de levure comprenant un YAC de $670 \mathrm{~kb}$ portant le gène de l'HGPRT (hypoxanthine guanylphosphoribosyl transférase) avec des cellules souches embryonnaires ES déficientes en HGPRT. Les cellules ES HGPRT + ont été alors sélectionnées sur milieu sélectif et injectées dans les blastocystes. Jakobovits et al. démontrent que ces cellules ES possédant maintenant un YAC participent pleinement au développement de la souris et, notamment, de sa lignée germinale, permettant d'obtenir des animaux hétérozygotes possédant le transgène [1].

A. Schedl et al. de l'équipe de Günther Schütz, utilisèrent, quant à eux, la technique classique de l'injection d'ADN, ici un chromosome artificiel de levure de $250 \mathrm{~kb}$ portant le gène de la tyrosinase, dans des ovocytes fécondés de souris albinos. Ces auteurs utilisèrent une méthode particulière leur permettant d'augmenter beaucoup la quantité d'ADN de YAC préparé par électrophorèse à partir des levures recombinées [2]. La fréquence d'obtention des souris transgéniques corrigées de leur caractère albinos du fait d'une expression normale de la tyrosinase fut tout à fait similaire à celles d'expériences de transgénèse avec des fragments d'ADN de petite taille. La tyrosinase transgénique s'exprimait avec exactement la même spécificité que l'enzyme endogène de sujets normaux, dans toutes les lignées obtenues, sans aucun effet de position : chez ces animaux, le niveau d'accumulation du messager était directement proportionnel aux nombres de copies intégrées et ne dépendait pas du site d'intégration [2]. Strauss et al. [3], enfin, rapportent la transmission dans la lignée germinale d'un YAC de $150 \mathrm{~kb}$ contenant le locus murin codant pour le collagène $\alpha_{1}$ (I), introduit par lipofection dans des cellules ES. Dans ces trois articles, les YAC ne semblaient pas avoir subi d'importants réarrangements malgré leur taille. Une conséquence potentiellement désavantageuse de la technique utilisée par Jakobovits et al., la fusion de sphéroplastes de levures, c'est-à-dire de levures dont la membrane externe a été digérée et qui sont limités à leur membrane plasmique, avec des cellules ES est que cela aboutit au transfert de la totalité du matériel génétique de levure qui est retrouvé chez les animaux et est transmis dans la lignée germinale. Dans un quatrième article, qui doit paraître prochainement dans Nature Genetics, une autre équipe a, comme les chercheurs du laboratoire de R. Jaenisch [3], utilisé la lipofection des cellules ES pour y faire pénétrer un YAC purifié contenant, dans ce cas, un gène de chaîne lourde d'immunoglobuline [4]. Les applications de ces techniques sont probablement multiples. C'est de plus en plus fréquemment que l'on isole un gène candidat de maladie sur un YAC. Le transfert de ce YAC dans une souris dont le phénotype constitue un modèle de la maladie étudiée pourrait permettre de confirmer la responsabilité du gène isolé, ainsi que cela a été fait avec le gène de tyrosinase transféré chez les souris albinos. Comme cela est discuté plus haut, des séquences portées par un YAC peuvent être facilement modifiées dans la levure par recombinaison homologue. Combinée éventuellement avec le knock-out de gènes endogènes de souris, cette méthode devrait permettre une étude in vivo des relations structure/fonction d'un gène donné. La possibilité de transférer de grands loci, par exemple contenant dans leur configuration germinale des gènes d'immunoglobulines ou des récepteurs $\mathrm{T}$ des lymphocytes, pourrait également constituer un excellent moyen d'en étudier les réarrangements somatiques ultérieurs. Dans tous ces cas, l'étude devrait être facilitée par l'intégration de tout un domaine chromatinien, possédant la totalité des séquences de régulation des gènes considérés qui devraient ainsi s'exprimer de manière autonome, indépendamment des sites d'intégration dans le génome des souris transgéniques.

A.K.

1. Jakobovits A, Moore AL, Green LL, Vergara GJ, Maynard-Curie CE, Austin HA, Klapholz S. Germ-line transmission and expression of a human-derived yeast artificial chromosome. Nature 1993 ; 362 : 255-8.

2. Schedl A, Montoliu L, Kelsey G, Schütz G. A yeast artificial chromosome covering the tyrosinase gene confers copy number-dependent expression in transgenic mice. Nature 1993 ; 362 : 205-6

3. Strauss WM, Dausman J, Beard C, Johnson C, Lawrence JB, Jaenisch R. Germ line transmission of a yeast artificial chromosome spanning the murine $\alpha_{1}$ (I) collagen locus. Science 1993 ; 259 : 1904-7. 